\title{
Tecnologia na aula de matemática: a importância do potencial semiótico
}

\author{
Vandoir Stormowski - PUCRS - vandoir@yahoo.com.br \\ Maria Alice Gravina - UFRGS - gravina@mat.ufrgs.br \\ José Valdeni de Lima - UFRGS - valdeni@inf.ufrgs.br
}

Resumo: Este artigo tem o objetivo de analisar a importância da identificação, por parte dos professores de matemática, do potencial semiótico dos softwares de geometria dinâmica. A partir de uma revisão bibliográfica, apresenta a relevância dos diferentes registros de representação semiótica para a aprendizagem em matemática, e contempla as implicações da abordagem instrumental quando da inserção dos recursos tecnológicos digitais na sala de aula. É a capacidade de relacionar conceitos matemáticos (identificáveis no software) com signos e significados que emergem do uso da ferramenta ou da realização da tarefa, que caracterizam o potencial semiótico dos softwares de geometria dinâmica. $\mathrm{O}$ estudo conclui que, para uma incorporação efetiva dos recursos tecnológicos no planejamento das aulas de matemática, os professores precisam identificar previamente o potencial semiótico da ferramenta a ser utilizada. Deste modo, a formação de professores de matemática deve capacitar tal identificação.

Palavras-chave: potencial semiótico; professores; geometria dinâmica.

\section{Technology in the mathematics classroom: the importance of semiotic potential}

\begin{abstract}
This article aims to analyze the importance of identifying, by mathematics teachers, semiotic potential of the dynamic geometry software. From a landscape review, presents the relevance of the different registers of semiotic representation for learning in mathematics, and considers the implications of the instrumental approach when inserting digital technologies in the classroom. It is the ability to relate mathematical concepts (identifiable in software) with signs and meanings that emerge from the use of the tool or task performance, featuring the semiotic potential of the dynamic geometry software. The study concludes that for an effective incorporation of technological resources in planning mathematics lessons, teachers need to identify in advance the potential of semiotic tool to be used. Thus, the mathematics teacher's education should enable such identification.
\end{abstract}

Keywords: semiotic potential; teachers; dynamic geometry.

\section{Introdução}

A importância da tecnologia no desenvolvimento da matemática é evidenciada em diversas situações em que seu uso promove mudanças substanciais na forma de ver e fazer matemática. Exemplos disso são ferramentas materiais e simbólicas, tais como, o desenvolvimento do sistema decimal, as tábuas logarítmicas (Artigue, 2002), e mais recentemente, o desenvolvimento dos recursos computacionais com Sistemas de Computação Algébrica, Softwares de Análise Numérica, Simuladores de Sistemas Dinâmicos, softwares de geometria e outros. No entanto, segundo a autora, apesar do 
amplo uso destes recursos por outros profissionais (matemáticos, engenheiros, geólogos, etc), o contexto escolar ainda se caracteriza por aulas expositivas e de repetição de procedimentos, de modo que a tecnologia para a sala de aula ainda é subutilizada.

Em uma análise de estudos internacionais na área, Joubert (2013) destaca que as tecnologias digitais são utilizadas de forma insuficiente nas aulas de matemática, e quando utilizadas, seu potencial é pouco explorado. Neste sentido, a autora afirma que apenas fornecer tecnologia para escola não é suficiente, e que o ponto crucial é a análise do papel do professor no contexto escolar tecnológico, de modo que a formação de professores para o uso da tecnologia possui significativa importância.

Em pesquisa (Barbosa, 2012) realizada pelo Comitê Gestor da Internet no Brasil (CGI.br) envolvendo alunos, professores e coordenadores de escolas públicas e privadas de áreas urbanas de todo o Brasil relativa à inserção das Tecnologias da Informação e Comunicação (TIC) na educação ${ }^{1}$, constatou-se a deficiência da formação de professores quanto ao uso destes recursos nas salas de aula. Verificou-se que cerca de metade dos professores já realizou curso específico para capacitação no uso de recursos tecnológicos, e mesmo assim, dois terços dos professores consideram que os alunos dominam melhor estes recursos do que eles próprios. Apesar dos investimentos em capacitação docente, os dados "mostram que os resultados desses esforços ainda são pouco efetivos" (Barbosa, 2012, p. 30), considerando que a sala de aula ainda se caracteriza essencialmente por aula expositiva, exercícios de fixação de conteúdo e interpretação de texto. O mesmo estudo ainda aponta que inclusive a formação básica dos professores mais jovens parece explorar insuficientemente estes recursos na prática pedagógica, e que atividades que incorporem as TIC no processo educacional ainda são pouco frequentes tanto em escolas públicas quanto privadas.

Tendo caracterizada a importância do professor no processo de incorporação das tecnologias digitais nas aulas de matemática, este artigo se debruça sobre um dos aspectos centrais do fazer docente: o planejamento didático de atividades que envolvam recursos digitais. Mais especificamente, aborda a relevância da análise do potencial semiótico (Mariotti, 2012) destas ferramentas, e as implicações no planejamento de atividades didáticas pertinentes.

Deste modo, a partir de uma revisão bibliográfica, o objetivo do artigo é enfatizar a importância da identificação do potencial semiótico de ferramentas tecnológicas, de modo a garantir que o planejamento destas atividades didáticas propicie o uso eficiente destes recursos no processo de aprendizagem matemática.

Existe grande diversidade de recursos digitais para o ensino de matemática. Com o intuito de delimitar melhor esta análise, se optou pelo enfoque nos softwares de Geometria Dinâmica (GD) tais como Cabri, GeoGebra, Cinderella entre outros. Os ambientes de GD são contemplados em diversas pesquisas por apresentarem características que possibilitem enriquecer a prática pedagógica relativa à geometria em sala de aula (Leung e Lee, 2013). E este diferencial, faz com que a GD tenha relevância no que se refere à incorporação de recursos tecnológicos em sala de aula.

De forma sintética, o potencial semiótico de uma ferramenta se caracteriza pela possibilidade ou capacidade que a mesma possui em articular conceitos matemáticos culturalmente estabelecidos, com "conceitos" particulares que cada sujeito desenvolve no uso destas ferramentas ou na realização de tarefas específicas com apoio das mesmas (Bussi e Mariotti, 2008). 
Para viabilizar a análise do potencial semiótico no ensino de matemática com apoio de recursos tecnológicos, duas articulações teóricas são necessárias. Por um lado é preciso analisar a relação entre sujeito e ferramenta, e a conseqüente elaboração de esquemas de uso, e deste modo a Abordagem Instrumental proposta por Rabardel (1995) tem papel importante. Por outro lado, é necessário entender como ocorre o registro do conhecimento matemático identificável nas ferramentas, e que a aprendizagem matemática somente ocorre com a mobilização de diferentes registros de representação (Duval, 2009).

Estas duas abordagens são apresentadas nas seções 2 e 3 deste artigo, respectivamente. A seção 4 analisa o potencial semiótico e suas implicações no fazer docente, e as conclusões e considerações finais são apresentadas na última seção.

\section{Atividades com instrumentos}

A Abordagem Instrumental (Rabardel, 1995) fornece elementos "teóricos apropriados para o estudo da ação do sujeito, mediado por um instrumento" (Bittar, 2011 , p. 160) tal como o uso de softwares pelo professor, bem como o a análise de situações de ensino e aprendizagem tanto presencial como a distância.

Nela, o processo de apropriação dos artefatos também modifica o sujeito, tanto em termos cognitivos como comportamentais. A Abordagem Instrumental analisa sujeito e artefato, com atenção especial aos esquemas de utilização necessários para realizar a ação. Segundo Rabardel (1995), o sujeito é o indivíduo que realiza a ação, e o artefato é um dispositivo material ou simbólico (martelo, lápis, computador, software, gráfico, etc) ao qual são associados esquemas de utilização (padrões invariantes de usos para classes de situações). Os esquemas são fruto de situações experimentadas pelo indivíduo, e que vivendo em sociedade também recebe influências culturais ou sociais relacionadas ao uso do mesmo.

$\mathrm{Na}$ Abordagem Instrumental é necessário diferenciar artefato de instrumento. $\mathrm{O}$ artefato se transforma progressivamente em instrumento, quando a ele são associados esquemas de utilização. Esta transformação ocorre tanto na adaptação de esquemas familiares pré-existentes quanto na criação de novos esquemas pelo sujeito. Este processo de transformação do artefato em instrumento é chamado de Gênese Instrumental (Artigue, 2002; Trouche, 2004; Rabardel, 1995).

Desta forma, o instrumento não é entendido apenas pelas suas características técnicas, mas como um elemento que leva em consideração o sujeito que o utiliza. O instrumento é próprio de cada sujeito, pois um mesmo artefato se transforma em um instrumento diferente para cada sujeito, dado que cada indivíduo associa a ele esquemas de utilização particulares, oriundos e consequência de experiências ou conhecimento específico de cada um. A denominação de instrumento está associada à forma aprimorada com que cada sujeito utiliza a ferramenta. É na realização de tarefas com a ferramenta, que esquemas particulares de utilização são elaborados e adaptados, e emergem signos $^{2}$ pessoais ou particulares. São estes signos pessoais que são parte importante da análise do potencial semiótico a ser realizado.

Ou seja, a Gênese Instrumental é o processo pelo qual o artefato se transforma em instrumento, quando a ele são agregados funções e esquemas de utilização pelo sujeito, permitindo que o artefato seja apropriado e integrado às suas práticas. Quando se trata do processo de apropriação de tecnologia por parte de docentes, "o processo de gênese instrumental é particularmente complexo, pois os artefatos tornam-se 
instrumentos não só nas práticas matemáticas do professores, mas também em suas práticas didáticas" (Fuglestad et al., 2010, p. 296). Para especificar esta complexidade, pode se considerar uma cascata de processos de gênese instrumental (Gravina et al., 2013) relacionada à apropriação do recurso tecnológico pelo professor de matemática: primeiramente ocorre a gênese relativa à aprendizagem matemática do professor, onde o software se transforma em instrumento para explorar conceitos matemáticos, sendo mediador na relação do sujeito com os objetos matemáticos, promovendo a aprendizagem; em seguida, o artefato se transforma em instrumento didático para professor, onde as características, limitações e potencialidades pedagógicas do software se evidenciam; em um terceiro momento, há a expectativa de que o professor consiga promover o processo de gênese instrumental com seus alunos em sala de aula, de modo que estes possam se utilizar do aplicativo para aprender matemática.

A transformação de um artefato em instrumento por si só já caracteriza a gênese instrumental com "uma evolução contínua, não-trivial e demorada" (Drijvers et al., 2010, p. 108), de modo que esta complexidade aumenta substancialmente considerando as diferentes gêneses colocadas em cascata na formação de professores, fazendo com que o professor precise de "muitas experiências com o artefato" (Gravina et al., 2013) para a efetiva apropriação deste recurso.

\section{Registros de representação semiótica e aprendizagem matemática}

Na matemática se necessita de sistemas de representação ou expressão além da linguagem natural e das imagens, comuns em outras áreas de conhecimento. São necessários diferentes sistemas de numeração, notações algébricas e geométricas, gráficos, símbolos, diagramas, esquemas, constituindo por si só uma rede de representações particular e complexa.

Considerando as representações semióticas como produções constituídas de signos (língua natural, fórmula, símbolo, gráfico, figura,...), é importante perceber que as mesmas não são utilizadas apenas para comunicação ou registro, mas são fundamentais na atividade matemática. Dada a característica de que os objetos de estudo da matemática são entes abstratos, a única forma de acessar e tratar estes entes é através dos signos que os representam, ou seja, da representação semiótica utilizada.

O objeto e sua representação são distintos, ao ponto que uma representação semiótica de um objeto é sempre uma representação parcial do mesmo, dadas as características e possibilidades de mobilização que evidencia (Duval, 2009). Por exemplo, a representação gráfica de uma função evidencia características diferentes que a representação algébrica da mesma, além de permitirem abordagens distintas. Ao mesmo tempo, não é possível ter compreensão em matemática quando não se distingue o objeto de sua representação, dado que sempre é uma representação parcial do mesmo.

Relacionando aprendizagem com as representações semióticas em matemática, Duval afirma que "não há noesis sem semiosis" (2009, p. 27). Ou seja, que não há apreensão conceitual de um objeto sem a utilização de representações semióticas.

Devido a grande variedade de representações semióticas utilizadas em matemática, Duval utiliza o termo registro para designar diferentes tipos destas representações, e que em termos amplos são assim classificados: língua natural (argumentação), sistemas de escritas (numérica, algébrica e simbólica), figuras geométricas e gráficos. 
No caso da mudança de representação, ou na transformação de uma representação semiótica em outra, se observa dois tipos que são bem diferentes (Duval, 2009): tratamento e conversão. O tratamento é a mudança de representação dentro de um mesmo registro, tal como a mudança de uma equação para um sistema de equações. A conversão envolve a mudança de registro para outro, tal como a expressão algébrica de uma função para a sua representação gráfica

Por mais que matematicamente seja considerada uma atividade lateral e evidente, cognitivamente a conversão é a transformação que conduz aos mecanismos subjacentes á compressão, e por isso deve ter atenção especial nas pesquisas em didática e ensino de matemática (Duval, 2003).

Considerando que o único acesso aos objetos matemáticos é pela representação semiótica, que cada representação evidencia particularidades distintas do objeto, e que não se pode confundir o objeto com o conteúdo de sua representação, Duval conclui que a "articulação dos registros constitui uma condição de acesso à compreensão em matemática" (2003, p. 21). O autor afirma que é a partir da coordenação de diversas representações semióticas de um mesmo objeto, que se tem acesso ao efetivo conceito do objeto considerado, e que a compreensão integral ocorre com a coordenação de registros que se manifesta com a velocidade e espontaneidade da atividade cognitiva de conversão. Ou seja, para que ocorra aprendizagem em matemática há a necessidade da coordenação de diversos registros de representação semiótica.

Os diferentes softwares de GD podem contribuir muito para explorar a transformação de conversão na sala de aula, dado que possibilitam a representação de um mesmo objeto, com diferentes registros de representação. Veja a representação de alguns elementos sob diferentes registros no GeoGebra:

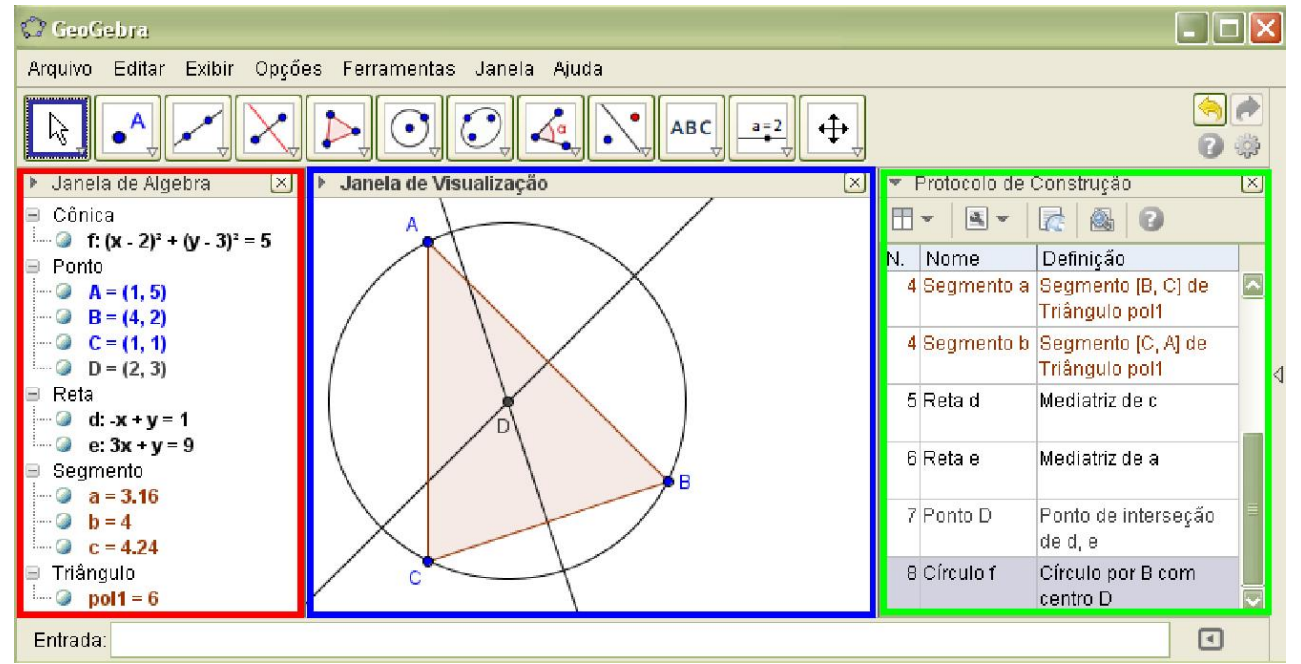

Figura 1: interface do GeoGebra.

$\mathrm{Na}$ figura acima temos um triângulo e seu circuncentro representados geometricamente (destaque azul), algebricamente (destaque vermelho) e com língua natural (destaque verde). Neste caso, além de permitir a representação, o software de GD permite a interação sobre o objeto matemático através da representação geométrica ou algébrica. Este é um fato importante, pois privilegia os dois sentidos da transformação de conversão, permitindo uma compreensão mais aprofundada do objeto matemático em questão. 


\section{Potencial semiótico dos softwares de Geometria Dinâmica}

A mediação se caracteriza pela intervenção de um elemento intermediário numa relação, e esta deixa de ser direta e passa a ser mediada por este elemento. Para Vygotsky, a relação do homem com mundo se dá pela mediação de signos e artefatos (Oliveira, 2002). E é neste contexto que é desenvolvida a Teoria da Mediação Semiótica (TMS) por Bussi e Mariotti (2008) que, do ponto de vista didático, analisa os diferentes tipos de signos envolvidos em atividades realizadas com apoio de artefatos. A TMS reconhece o papel central dos signos em atividades de ensino-aprendizagem, e segundo Mariotti (2012), a evolução de significados, que se originam desta interação complexa de signos, pode ser descrita no desenvolvimento do processo de mediação semiótica. "Promover e orientar este processo é uma questão crucial e uma tarefa exigente para o professor" (Mariotti, 2012, p. 27).

Segundo a autora, a mediação ocorre quando há um conteúdo matemático a ser mediado pelo professor para o aluno. Neste processo, quando um artefato é utilizado intencionalmente para promover esta mediação, a análise prévia do potencial semiótico do artefato é crucial para que o professor consiga articular adequadamente a atividade planejada, e que este planejamento propicie efetiva aprendizagem.

No processo de aprendizagem, onde os artefatos possuem papel central, estão envolvidos os significados e esquemas pessoais de cada indivíduo (aluno) na utilização do artefato para a realização de alguma atividade específica. Ao mesmo tempo, significados matemáticos podem estar relacionados ao artefato e sua utilização. "Esta dupla relação semiótica será chamada de potencial semiótico do artefato" (Bussi e Mariotti, 2008, p. 754). Ou seja, são as potencialidades que o artefato possui para articular significados pessoais e significados matemáticos no processo de aprendizagem matemática.

$\mathrm{Na}$ utilização de artefatos para promover conhecimento matemático, Mariotti (2013) destaca que é preciso distinguir dois tipos de significados matemáticos: evocados e emergentes. Considerando atividades com o ábaco, tal como exemplo apresentado por Bussi e Mariotti (2008), os significados evocados são as relações com o sistema de numeração decimal, posicional, e todas as relações com conhecimento matemático que este material pode oferecer, e reconhecido pelo professor. Ou seja, são tanto os significados matemáticos contemplados no artefato quando de sua criação, quanto aqueles que o professor articula em atividades com o artefato, mesmo que não tenham sido previstos pelo desenvolvedor. $\mathrm{E}$ os significados que o aluno vai atribuindo e identificando nas atividades com o ábaco, são os significados emergentes, provenientes dos esquemas particulares que são mobilizados para a realização da atividade ou relacionados ao uso do próprio artefato.

Portanto, o potencial semiótico do artefato se encontra na relação do mesmo com os significados pessoais que emergem das atividades instrumentadas, e na relação com os conhecimentos evocados pelo seu uso, que são reconhecidos como matemática pelo especialista (Mariotti, 2013), que neste caso é o professor por ser representante do conhecimento matemático culturalmente estabelecido.

Considerando o potencial semiótico, cada artefato possui um papel importante no planejamento de atividades didática, desde que este potencial seja identificado previamente pelo professor e considerado no planejamento. Segundo Mariotti (2009), mesmo que esta identificação seja uma tarefa difícil, constitui um elemento básico para o desenvolvimento de qualquer plano pedagógico que envolva artefatos. 
É fundamental e indispensável, portanto, que se identifique previamente o potencial semiótico daquele artefato, para apenas depois se colocar no planejamento de atividades com seu uso. Para identificar este potencial é necessário analisar atividades em que o artefato é utilizado para realizar determinada tarefa, e pode envolver diferentes perspectivas: cognitiva, epistemológica, etc. No caso de artefatos tecnológicos com softwares de GD, um ponto de partida são as funcionalidades didáticas projetadas pelos desenvolvedores, mas como são muitos os caminhos que se abrem na realização de uma atividade, este é apenas o primeiro passo (Mariotti, 2013).

Os softwares de GD se caracterizam por apresentarem régua e compasso virtuais (Gravina, 2001). Atualmente, alguns destes softwares incorporaram outras funcionalidades, de modo a se constituírem ferramentas bastante completas para articulação de diversos ramos de conhecimento matemático, de modo que se constituem um bom ponto de partida para análise da articulação de recursos tecnológicos no ensino de matemática. Deste modo, embora se possa escolher os mais diferentes recursos digitais disponíveis para ensino de matemática, a opção pela GD passa a ser um ponto de partida pertinente para a análise do potencial semiótico.

$\mathrm{Na}$ bibliografia encontramos exemplos sobre a forma de identificação do potencial semiótico. Uma dessas situações é apresentada por Falcade et al. (2007) quando da análise de uma relação entre pontos representados em um software de GD chamado Cabri. O referido exemplo envolvia uma atividade em que os alunos movimentavam um determinado ponto pela tela do Cabri, e em consequência, outro ponto se movimentava de forma associada representando uma reta.

Algo semelhante pode ser ilustrado na seguinte situação: o movimento do ponto $\mathrm{P}$ faz com que o ponto $\mathrm{B}$ associado, descreva uma circunferência com centro em $\mathrm{A}$ e de raio $\mathrm{AB}$. A figura abaixo, ilustra esta situação no GeoGebra.

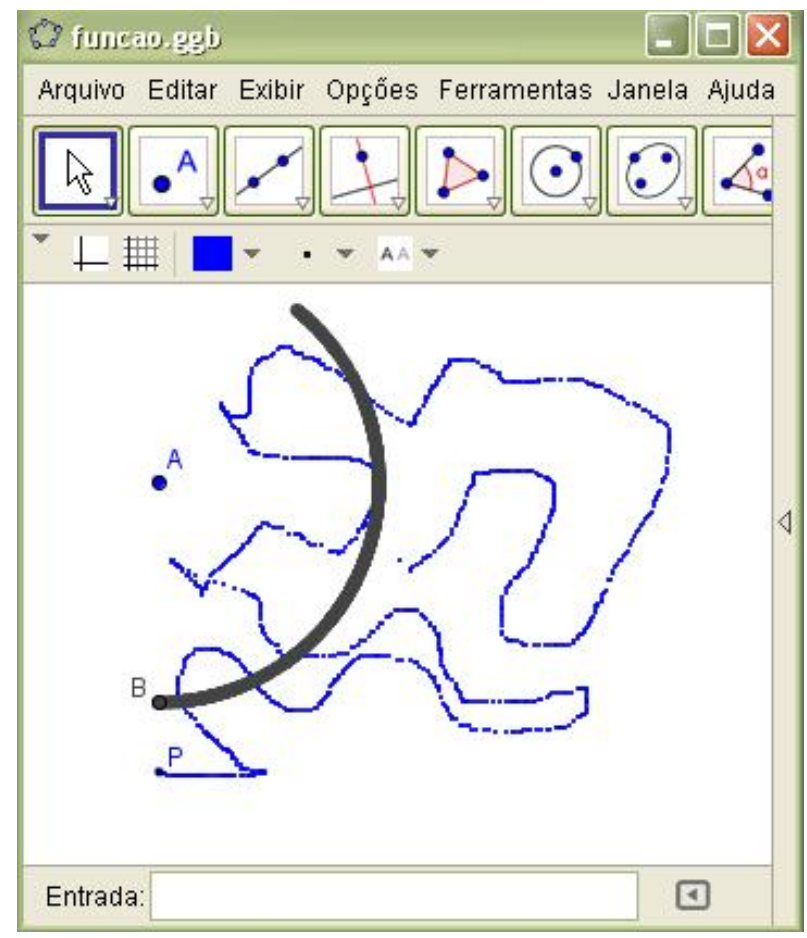

Figura 2: atividade no GeoGebra. 
Para identificar o potencial semiótico do software de GD neste caso, é necessário identificar:

- os conceitos matemáticos relacionados ao artefato ou com a atividade: neste caso, os pontos $\mathrm{B}$ e $\mathrm{P}$ estão associados através de uma relação especial de dependência, ou seja, a posição do ponto B depende da posição ocupada pelo ponto $\mathrm{P}$. Deste modo, temos uma função (estabelecida geometricamente) entre os referidos pontos, onde $\mathrm{P}$ se caracteriza como elemento independente da relação e B o elemento dependente.

- os signos particulares que emergem do uso do artefato pelo sujeito: estes signos são pessoais de cada aluno, de modo que sua identificação só é possível através da comunicação (verbal ou escrita) entre professor e aluno. No entanto, quando da necessidade de se identificar estes signos antes da realização da atividade, o professor precisa estabelecer quais os possíveis signos mencionados ou referidos pelos alunos, e que podem ser vinculados aos conceitos matemáticos em questão.

No exemplo apresentado em Falcade et al. (2007), as autoras analisaram uma extensa sequência com transcrição do diálogo estabelecido por professor-aluno, alunoaluno, e no registro escrito de atividades.

Como esta identificação não é um processo simples e imediato (Mariotti, 2013), envolverá a experiência do professor com o artefato, com os alunos, e até mesmo com atividades similares.

\section{Considerações finais}

Dado o cenário inicialmente apresentado, em que a sala de aula de matemática ainda não incorpora adequadamente as tecnologias digitais disponíveis, se evidencia que uma análise da relação do professor com os artefatos tecnológicos é necessária. Neste sentido, tanto a Abordagem Instrumental que analisa a relação do sujeito com o artefato, quanto a forma peculiar de representação do conhecimento matemático, possuem papel importante.

O potencial semiótico dos softwares de GD, estabelece exatamente esta relação entre os signos que emergem da relação com o artefato e aqueles conceitos estabelecidos culturalmente como conhecimento matemático.

Neste contexto, a apropriação do recurso tecnológico pelo professor, para que possa ser utilizado adequadamente em seus planejamentos didáticos, é um processo complexo e com diferentes fases.

Por um lado, o professor precisa se apropriar do artefato, relacionando a ele esquemas de utilização particulares, de modo que com o processo de Gênese Instrumental, o artefato se transforme em instrumento. No entanto, para que possa ser utilizado com eficiência em sala de aula, outra etapa se faz necessária, a transformação do instrumento em instrumento de mediação semiótica, quando o professor explora o potencial semiótico do referido artefato, e para que desta forma o mesmo possa promover o processo de mediação semiótica envolvido na tarefa de ensinar. 


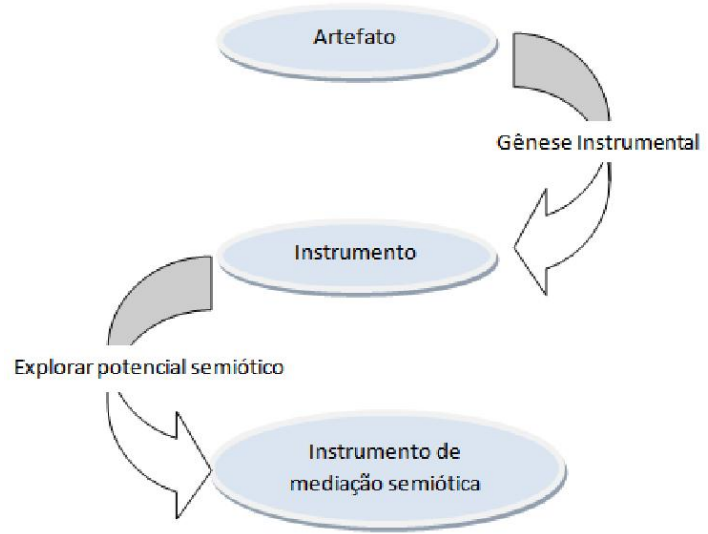

Figura 3: explorar o potencial semiótico.

Como consequência desta análise, se considera que a formação dos professores de matemática para uso da tecnologia deve contemplar todos estes aspectos apresentados, e que principalmente contemple a exploração do potencial semiótico dos softwares (ou outros recursos) utilizados no processo de ensino-aprendizagem.

1 A pesquisa completa é apresentada por Barbosa (2012), e busca identificar o quanto as TIC estão inseridas no processo educacional das escolas brasileiras, é realizada anualmente. Disponível em: $<$ http://op.ceptro.br/cgi-bin/cetic/tic-educacao-2011.pdf>. Acesso em: 10 ago. 2013.

${ }^{2}$ Neste texto o termo signo se refere à união indissolúvel entre significado e significante, tal como na abordagem européia da semiótica.

\section{Referências}

ARTIGUE, M. Learning mathematics in a CAS environment: the Genesis of a reflection about instrumentation and the dialectics between technical and conceptual work. International Journal of Computers for Mathematical Learning, Netherlands, n. 7. p. 245-274, 2002.

BARBOSA, A. (Org.). Pesquisa sobre o uso das tecnologias da informação e comunicação no Brasil: tic educação 2011. São Paulo: Comitê Gestor da Internet no Brasil, 2012.

BITTAR, M. A abordagem instrumental para o estudo da integração da tecnologia na prática pedagógica do professor de matemática. Educar em revista, Curitiba, p. 157-171, 2011.

BUSSI, M. G. B.; MARIOTTI, M. A. Semiotic mediation in the mathematics classroom: artifacts and signs after a vygotskian perspective. Handbook of international research in mathematics education, New York, p. 746-783, 2008.

DRIJVERS, P.; KIERA, C.; MARIOTTI, M. A.; AINLEY, J.; ANDRESSEN, M.; CHAN, Y. C.; DANA-PICARD, T.; GUEUDET, G.; KIDRON, I.; LEUNG, A.; MEAGHER, M. Integrating Technology into Mathematics Education: theoretical perspectives. In: HOYLES, C.; LAGRANGE, J. (Org.). Mathematics Education and Technology-Rethinking the Terrain. Springer US, 2010. p. 89-132. 
DUVAL, R. Registros de representações semióticas e funcionamento cognitivo da compreensão em matemática. In: Aprendizagem em matemática: registros de representação semiótica. Campinas: Papirus, 2003. p. 11-34.

DUVAL, R. Semiósis e Pensamento Humano: registros semióticos e aprendizagens intelectuais. São Paulo: Livraria da Física, 2009.

FALCADE, R.; LABORDE, C.; MARIOTTI, M. A. Approaching functions: cabri tools as instruments of semiotic mediation. Educational Studies in Mathematics, vol. 66, p. 317-333, 2007.

FUGLESTAD, A. B.; KYNIGOS, L. H. C.; MONAGHAN, J. Working with teachers: context and culture. In: HOYLES, C.; LAGRANGE, J. (Org.) Mathematics Education and Technology-Rethinking the Terrain. Springer US, 2010. p. 293310.

GRAVINA, M. A. Os ambientes de geometria dinâmica e o pensamento hipotéticodedutivo. 2001. Tese (Doutorado em Informática na Educação) - PGIE/UFRGS, Porto Alegre.

GRAVINA, M. A.; BARRETO, M. M.; NOTARE, M. Continuing professional development and digital media in mathematics education. Congress of European Research in Mathematics Education, Turkey, Feb. 2013.

JOUBERT, M. Using digital technologies in mathematics teaching: developing an understanding of the landscape using three "grand challenge" themes. Educational Studies in Mathematics, vol. 82, n. 3, p. 341-359, mar. 2013.

LEUNG, E. L.; LEE, A. M. S. Student's geometrical perception on a task-based dynamic geometry platform. Educational Studies in Mathematics, vol. 82, n. 3, p. 361-377, mar. 2013.

MARIOTTI, M. A. Artifacts and signs after a Vygotskian perspective: the role of the teacher. ZDM Mathematics Education, vol. 41, p. 427-440, 2009.

MARIOTTI, M. A. ICT as opportunities software for teaching-learning in mathematics classroom: the semiotic potential of artefacts. In: TSO, T. Y. (Org.). Proceedings of the $36^{\text {th }}$ Conference of the International Group for the Psychology of Mathematics Education. Taipei, Taiwan: PME, 2012. vol. 1, p. 25.

NOTARE, M. R.; GRAVINA, M. A. A formação continuada de professores de matemática e a inserção de mídias na escola. Anais do VI Colóquio de História e Tecnologia no Ensino de Matemática (VI HTEM), São Carlos, 2013.

OLIVEIRA, M. K. Vygotsky: aprendizado e desenvolvimento, um processo sóciohistórico. 4.ed. São Paulo: Scipione, 2002.

RABARDEL, P. Les hommes et les technologies: aprproche cognitive des instruments contemporains. Paris: Armand Colin, 1995.

TROUCHE, L. Managing the complexity of human/machine interactions in computerized Learning Environments: guiding student's command process through instrumental orchestrations. International Journal of Computers for Mathematical Learning, Netherlands, n. 9, p. 281-307, 204. 